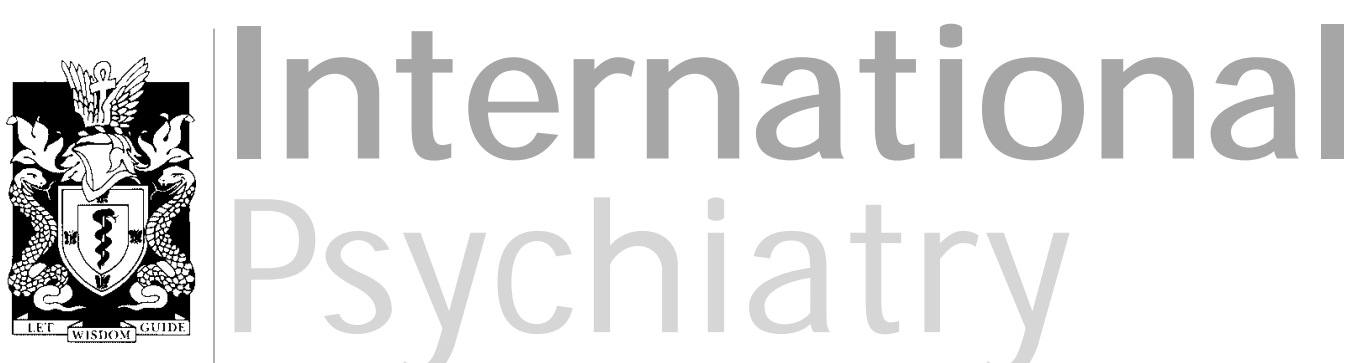

\title{
International Psychiatry - the way forward
}

\section{Hamid Ghodse}

Director, Board of International Affairs and Editor, International Psychiatry

\section{1}

II psychiatrists would acknowledge that communication between individuals is fundamental to their speciality, but it is perhaps less obvious that the increasing ease and speed of communication worldwide are also having a profound effect on psychiatric practice. With this improved communication comes an awareness of the commonality of many mental health issues and recognition that there is much to learn from others working in very diverse environments. It is also true that many mental health problems have an international dimension, particularly when large numbers of people are displaced by war or other disasters. Increasingly we need to have a better understanding of other cultures and the relationship between culture, mental health and psychiatric disorder.

Simultaneously there have been dramatic advances in many areas of psychiatry, including basic biomedical research, as well as behavioural and clinical research. There have been new discoveries in related fields, such as genetics, immunology and cellular function, that are already affecting psychiatric practice, and new techniques for investigating clinical processes are continually being devised. $\mathrm{H}$ ealth systems and health services are also the subject of research, into issues such as health needs, coverage of populations, utilisation, cost-effectiveness and the relationship with broader aspects of the health economy. In addition, there has been extensive reorganisation of health services, including mental health services, in many countries, and this has affected the training of health care professionals and changed the way in which health care is provided.

These changes and advances form the context for the launch of the Board of International Affairs (BIA) at the annual meeting of the Royal College in Cardiff in the summer of 2002. It was set up because of the growing recognition by the College that, in the 21st century, it needs to play a more prominent role on the international stage, by forming partnerships with similar bodies in other countries and by developing strong links with international organisations involved in mental health.
W ith members in many countries, the College has a firm base for this development, but it has acknowledged that it needs to improve its communication with members abroad and, more importantly, to develop a dialogue with them, and between them, to provide a forum for mutual learning with a genuinely international perspective. International Psychiatry is the bulletin of the BIA and its first issue signals this more open approach.

International Psychiatry provides an overview of current policy and practice in psychiatry in different countries and will help all mental health professionals to learn about and keep abreast of what is happening elsew here. This is valuable because the complex relationship between cultural variables and mental health differs from one country to another and this in turn affects treatment and attitudes to treatment. Learning about others' practices can act as a powerful but healthy challenge to entrenched assumptions. The bulletin's special features therefore include country profiles on mental health and mental health policy, which describe the structures for education, training, research, policy and practices, so that those who are interested in collaborative work, elective placements and so on have ready access to fundamental information. In addition, particular themes are explored in each issue from the perspective of different countries and international organisations, with commissioned reviews from experts on selected topics. The editors will actively search for sound and reputable work to provide readers with good information and there will be an emphasis on the policy and practice implications of new research. There will also be a section on forthcoming major events, worldwide. Information about the activities and working structures of the Royal College will be included so that members abroad can more easily identify with their College and know what is going on. There will be a correspondence section which, it is hoped, will foster multidisciplinary discussion and dialogue.

We hope you enjoy the first issue and feel encouraged to contribute to the future development of International Psychiatry.

\section{Contents}

Editorial

H amid Ghodse

Thematic papers terrorism

INTRODUCED BY

David H. Skuse

Daniel B. Herman and Ezra S. Susser

F. G. N jenga,

P. Kigamwa and

M. O konji

Joop de Jong, Ivan Komproe and Mark Van 0 mmeren 8

John, Lord

Alderdice

Country profiles

Malik Hussain

Mubbashar -

Pakistan

Alfredo Pemjean Chile

Marcos Pacheco de Toledo Ferraz and Elisaldo A. Carlini Brazil

Vikram Patel and Shekhar Saxena India

Associations and collaborations John Cox

News, notes, events 